\title{
纳米碳纤维的表面改性对水电解析氢反应催化活性的影响
}

\author{
钮东方 ${ }^{a}$ 丁勇 ${ }^{a}$ 马智兴 ${ }^{b}$ 王明辉 ${ }^{b}$ \\ 刘洲 ${ }^{b} \quad$ 张博文 ${ }^{b}$ 张新胜*,a \\ ( ${ }^{a}$ 华东理工大学化学工程联合国家重点实验室 上海 200237)

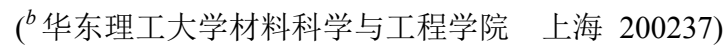

\begin{abstract}
摘要 主要研究纳米碳纤维(CNF)的表面改性对析氢反应(HER)催化活性的影响. 首先采用一种简单易行的超声处理 方法, 以混酸(浓硫酸和浓硝酸)为溶剂对 CNF 进行了表面化学处理, 以在其表面引入含氧(CNF-OX)官能团, 然后将 CNF-OX 在氨水中超声处理, 以引入含氮(CNF-ON)官能团, 以及将 CNF-OX 和硼酸混合经高温热解对 CNF-OX 进行掺 B 处理(B-CNF-OX). XPS 结果表明, 超声处理可以成功地在 CNF 表面引入含氧和含氮官能团, 嗍酸高温处理可以掺入 B 原子. 电化学测试结果表明, 经过表面改性后的 CNF 的 HER 催化性能都要好于未处理过的 CNF-UN, 其中 N 掺杂的 CNF-ON 表现出最好的 HER 催化活性, 且 CNF-OX 经掺 B 处理后, B-CNF-OX 的性能也较 CNF-OX 有所增强, 即三者 的催化活性大小如下: CNF-OX $<$ B-CNF-OX $<$ CNF-ON. 这说明 O、B、N 杂原子的掺杂会影响纳米碳纤维的析氢催化 活性，特别是 $\mathrm{N}$ 原子的掺入对纳米碳纤维 HER 催化活性有极大的促进作用.
\end{abstract}

关键词＼cjkstart纳米碳纤维; 析氢反应; 表面改性; 电催化性能; 电解水

\section{Effects of Surface Modification of Carbon Nanofibers on Their Electro- catalytic Activity for Hydrogen Evolution Reaction of Water Electrolysis}

Niu, Dongfang ${ }^{a} \quad$ Ding, Yong $^{a} \quad$ Ma, Zhixing ${ }^{b} \quad$ Wang, Minghui $^{b}$ Liu, Zhou ${ }^{b} \quad$ Zhang, Bowen ${ }^{b} \quad$ Zhang, Xinsheng*,a

$\left({ }^{a}\right.$ State Key Laboratory of Chemical Engineering, East China University of Science and Technology, Shanghai 200237)

( ${ }^{b}$ School of Materials Science and Engineering, East China University of Science and Technology, Shanghai 200237)

Abstract The effects of surface modification of CNF on their electrocatalytic activity for hydrogen evolution reaction (HER) were investigated. Firstly, Oxygen-containing functional groups were introduced onto the CNF surface (labeled as CNF-OX) by a simple sonochemical oxidation in mixed acids (concentrated sulfuric acid and nitric acid) and then the nitrogen-containing functional groups were introduced onto the CNF-OX surface (labeled as CNF-ON) by sonochemical treatment in ammonia, and the B-doped CNF was synthesized by mixing CNF-OX and boric acid in a ratio following by pyrolysis at $800{ }^{\circ} \mathrm{C}$ under $\mathrm{N}_{2}$ atmosphere (labeled as B-CNF-OX). The XPS results showed that the ultrasonic treatments could introduce oxygen- and nitrogen-containing functional groups onto the CNF surface successfully, the oxygen atoms content of CNF-OX was increased from $1.96 \%$ to $6.01 \%$ compared to the untreated CNF (CNF-UN), and nitrogen atoms content of CNF-ON was increased from $0 \%$ to $1.02 \%$ compared to CNF-OX. The pyrolysis of boric acid could introduce B element onto the CNF-OX surface where the B atoms content of B-CNF-OX was $1.00 \%$. The linear sweep voltammetry (LSV) test results showed that the HER electrocatalytic activity of the modified CNFs were higher than CNF-UN, and among them CNF-ON had the highest electrocatalytic activities for HER than the others, the onset overpotential of CNF-ON was $344 \mathrm{mV}$, which shifted $240 \mathrm{mV}$ positively compared to CNF-UN, and the electrocatalytic activity of B-CNF-OX was enhanced by B-doping treatment compared to CNF-OX. The Tafel test indicated CNF-ON had smallest Tafel slop of $154 \mathrm{mV} / \mathrm{dec}$ and largest exchange current density of $6.68 \mu \mathrm{A} / \mathrm{cm}^{2}$. The electrochemical impedance spectroscopy (EIS) was carried out to investigate the complex interfacial properties of the CNFs modified electrode, and the results showed that CNF-ON had the smallest charge transfer resistance of $1578 \Omega$ among all the catalysts, which means the nitrogen-doped CNF had higher activity than the oxygen- and boron-doped CNF. All above indicated that the doping of heteroatom $(\mathrm{O}, \mathrm{B}, \mathrm{N})$ onto the CNFs surface had effects on their electrocatalytic activity for HER, especially the introduction of $\mathrm{N}$ atom onto the CNF surface had excellent improvement.

Keywords carbon nanofibers; hydrogen evolution reaction; surface modification; electrocatalytic activity; water electrolysis

* E-mail: xszhang@ecust.edu.cn; Tel: 021-64250914

Received March 21, 2015; published May 20, 2015.

Supporting information for this article is available free of charge via the Internet at http://sioc-journal.cn.

Project supported by the National Natural Science Foundation of China (No. 21073061) and Shanghai university student innovative activities plan.

项目受国家自然科学基金(No. 21073061)和上海市大学生创新训练项目(No. S13028)资助. 


\section{1 引言}

氢气, 作为一种理想的燃料及能量载体由于其无污 染、高能量密度及易转化为其它能量形式等优点而越来 越受到人们的广泛关注 ${ }^{[1]}$. 而电解水制氢因其工艺简 单、产品纯度高且可以使用可再生能源来实现, 近年来 引起了研究者的广泛兴趣 ${ }^{[2]}$. 传统的析氢阴极材料(如 低碳钢) 由于其析氢过电位较高而使得电解水制氢能耗 较大, 因此, 寻求有效的阴极材料或催化剂来降低析氢 过电位成了析氢反应的研究重点. 纳米碳纤维作为一种 新型的碳材料, 由于具有独特的电子和结构特性及良好 的机械性能 ${ }^{[3 \sim 5]}$, 近年来在作为电催化剂和催化剂载体 材料方面受到了关注, 已被广泛应用于氧还原反应

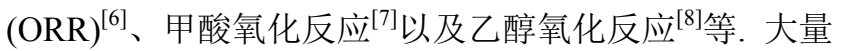

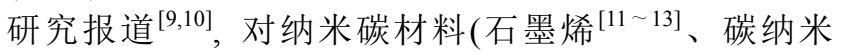
管 ${ }^{[14 ~ 16]}$ 等)进行表面改性, 在其表面引入一些杂原子(如 $\mathrm{O} 、 \mathrm{~N} 、 \mathrm{~S}$ )或和其它材料进行复合可以调整其电子特性, 使其电催化性能得到大幅提升, 但目前这些碳材料的表 面改性研究主要集中在 $\mathrm{ORR}^{[17,18]}$ 和质子交换膜燃料电 池(PEMFC) 催化剂等领域, 对于纳米碳纤维作为 HER 催化剂的表面改性研究还鲜有报道.

因此本文以纳米碳纤维为研究对象, 研究表面改性 对其作为 HER 催化剂性能的影响, 采用一种简单易行 的超声处理方法以及高温热处理法分别在纳米碳纤维 表面引入 $\mathrm{O} 、 \mathrm{~N}$ 和 $\mathrm{B}$ 原子, 考察了不同杂原子的掺杂对 纳米碳纤维在酸性溶液中析氢反应催化性能的影响.

\section{2 结果与讨论}

\subsection{CNFs 的 XPS 表征}

将不同处理方式的纳米碳纤维表面的 C, N, B 和 O 元素的 XPS 分析结果列于表 1 中. 从表 1 中可以看出, CNF-UN 表面的 N 和 B 元素含量为 0 , 而混酸超声氧化 后 CNF-OX 表面的 O 元素含量明显提高, 但仍未见有 $\mathrm{N}$ 元素的引入. 在氨水中超声处理后, CNF-OX 表面成功 地引入了 $\mathrm{N}$ 元素, 同时 CNF-OX 表面的 $\mathrm{O}$ 元素含量降 低, 含氧官能团的减少是由于 CNF-OX 表面的部分含氧 官能团被含氮官能团所取代. 对 CNF-OX 掺 B 处理后, 发现 B 元素含量从 $0 \%$ (CNF-OX)增加至 1.00\% (B-CNF-OX), 说明 $\mathrm{B}$ 原子成功地掺入到了 $\mathrm{CNF}$ 表面.

表 1 CNFs 表面元素组成的 XPS 分析结果

Table 1 XPS analytical results of surface element composition of CNFs

\begin{tabular}{lcccc}
\hline Sample & C (at.\%) & N (at.\%) & B (at.\%) & O (at.\%) \\
\hline CNF-UN & 98.04 & 0 & 0 & 1.96 \\
CNF-OX & 93.99 & 0 & 0 & 6.01 \\
CNF-ON & 94.67 & 1.02 & 0 & 4.31 \\
B-CNF-OX & 91.95 & 0 & 1.00 & 7.05 \\
\hline
\end{tabular}

由于含氧官能团的 CNFs 的 XPS 分峰拟合已在之前
的研究 ${ }^{[19]}$ 中有所讨论, 这里仅对 B-CNF-OX 和 CNF-ON 的 XPS 图谱进行分峰拟合分析. 首先对 B-CNF-OX 的 $\mathrm{C} 1 \mathrm{~s} 、 \mathrm{O} 1 \mathrm{~s}$ 和 B1s 峰进行窄扫, 并使用 XPSpeak 分峰软 件采用高斯函数对其作分峰处理, 如图 1 所示.
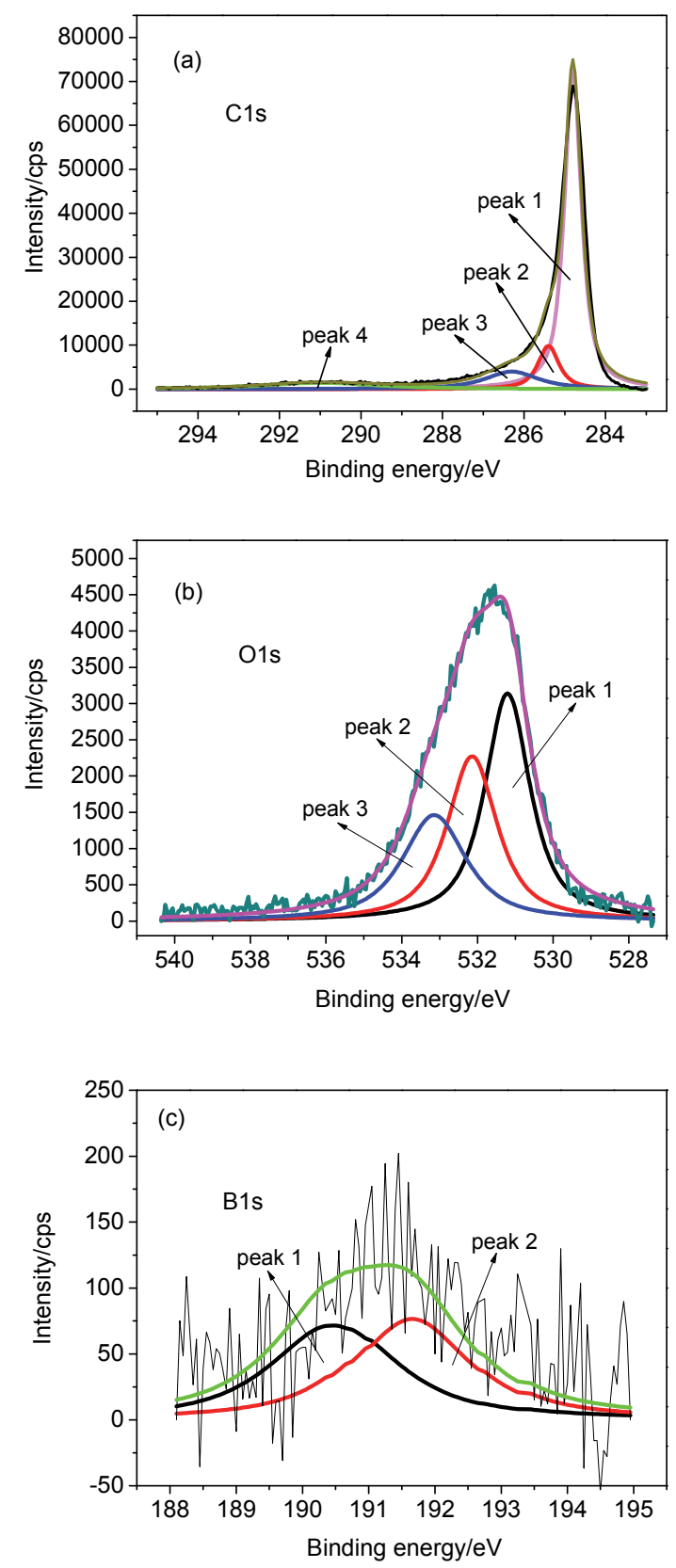

图 1 B-CNF-OX 的 XPS 图谱分峰结果. (a) C 1s, (b) O 1s, (c) B 1s Figure 1 Peak deconvolutions of the XPS spectra of B-CNF-OX. (a) $\mathrm{C}$ 1s, (b) O 1s, (c) B 1s

如图 1(a)所示, 这里将 B-CNF-OX 的 C1s 峰分成了 4 个峰 ${ }^{[19]}$, 主峰(peak 1)位于 $284.8 \mathrm{eV}$, 其主要是由 B-CNF-OX 表面的石墨层结构的碳原子组成; 第二个峰 (peak 2)位于 $285.4 \mathrm{eV}$, 可能是由 B-CNF-OX 中缺陷位 置的烃类 $\mathrm{C}-\mathrm{C}$ 键中 $\mathrm{C}$ 原子组成 ${ }^{[20]}$; 第三个峰(peak 3) 位于 $286.3 \mathrm{eV}$ ，其主要由 B-CNF-OX 表面醇和醚的 C- 
$\mathrm{O}$ 官能团组成; 第四个峰(peak 4)位于 $291.1 \mathrm{eV}$, 极有可 能是由吸附在 B-CNF-OX 微孔中的 $\mathrm{CO}_{2}$ 中 $\mathrm{C}$ 原子组成 的.

同样的, 对 O1s 峰也进行了如图 1(b)的分峰处理, 这里将 $\mathrm{O} 1 \mathrm{~s}$ 峰分成了 3 个峰 ${ }^{[21]}$, 其中 peak 1 位于 531.4 $\mathrm{eV}$, 主要是由羰基和醌基中 $\mathrm{C}=\mathrm{O}$ 键中 $\mathrm{O}$ 原子组成; peak 2 位于 $532.3 \mathrm{eV}$, 主要是由 B-CNF-UN 表面羟基 $\mathrm{C}-\mathrm{OH}$ 键中的 $\mathrm{O}$ 原子组成; peak 3 位于 $533.3 \mathrm{eV}$, 主要 是由 $\mathrm{C}-\mathrm{O}-\mathrm{C}$ 键中 $\mathrm{O}$ 原子组成. 最后对 $\mathrm{B}-\mathrm{CNF}-\mathrm{OX} \mathrm{B} 1 \mathrm{~s}$ 峰进行了如图 1(c)的分峰处理, 将其分成了两个峰 ${ }^{[15]}$, 其中 peak 1 位于 $191.3 \mathrm{eV}$, 归属于 $\mathrm{BC}_{2} \mathrm{O}$ 中 $\mathrm{B} 1 \mathrm{~s}$; peak 2 位于 $192.0 \mathrm{eV}$, 归属于 $\mathrm{BCO}_{2}$ 中 $\mathrm{B} 1 \mathrm{~s}$.

接着, 我们对 CNF-ON 中 $\mathrm{C} 1 \mathrm{~s} 、 \mathrm{O} 1 \mathrm{~s}$ 和 $\mathrm{N} 1 \mathrm{~s}$ 也进行 了类似的分峰处理, 由于其中 $\mathrm{C} 1 \mathrm{~s}$ 和 $\mathrm{O} 1 \mathrm{~s}$ 的分峰拟合结 果和上述 B-CNF-OX 相似, 因此这里就不再赘述. CNF-ON 中 N1s 的分峰结果如图 2 所示.

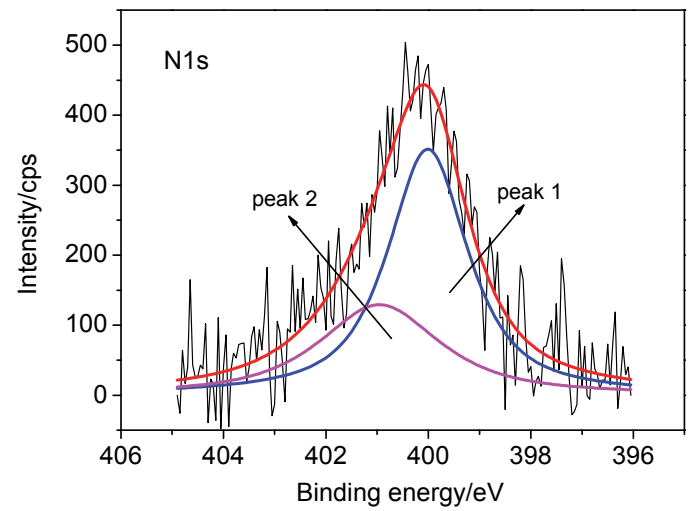

图 2 CNF-ON 的 XPS 图谱分峰结果, N1s

Figure 2 Peak deconvolutions of the XPS spectra of CNF-ON for N1s

从图 2 中可以看出, $\mathrm{N} 1 \mathrm{~s}$ 峰可以分成两个峰: peak 1 位于 $400.0 \mathrm{eV}$ ，归属于 $\mathrm{CNF}-\mathrm{ON}$ 表面的吡咯型氮; peak 2 位于 $400.9 \mathrm{eV}$, 归属于 $\mathrm{CNF}-\mathrm{ON}$ 表面的石墨层氮 ${ }^{[19]}$. 从 XPS 分析结果中我们可以发现 $\mathrm{N}$ 元素已经成功地掺杂 到 CNF 表面, 且主要是以吡咯型和石墨层氮的形式掺 入.

\subsection{CNFs 的线性扫描伏安曲线}

图 3 是几种制备的催化剂修饰电极在氮气饱和的 $0.5 \mathrm{~mol} / \mathrm{L} \mathrm{H}_{2} \mathrm{SO}_{4}$ 溶液中的线性扫描伏安(LSV)曲线图.

图 3 中可以看到, 超声氧化处理制得的 CNF-OX 和 氨水处理的 CNF-ON 相比未处理的 CNF-UN, HER 催化 活性均有所提高, CNF-UN、CNF-OX、CNF-ON 三者的 析氢起始过电位分别为 $581 、 501 、 344 \mathrm{mV}$, 可以发现掺 $\mathrm{N}$ 的 CNF-ON 起始过电位相比 CNF-UN 正移了 $240 \mathrm{mV}$ 左右, 而含氧的 CNF-OX 相比之下只正移 $80 \mathrm{mV}$, 说明 $\mathrm{N}$ 原子的掺入对 HER 催化活性有较大的增强作用, 这 可归因于氮原子在 $\mathrm{CNF}$ 表面主要是以吡咯型氮和石墨 层氮的形式掺入, 这些 $\mathrm{N}$ 原子的引入势必会调整其周围

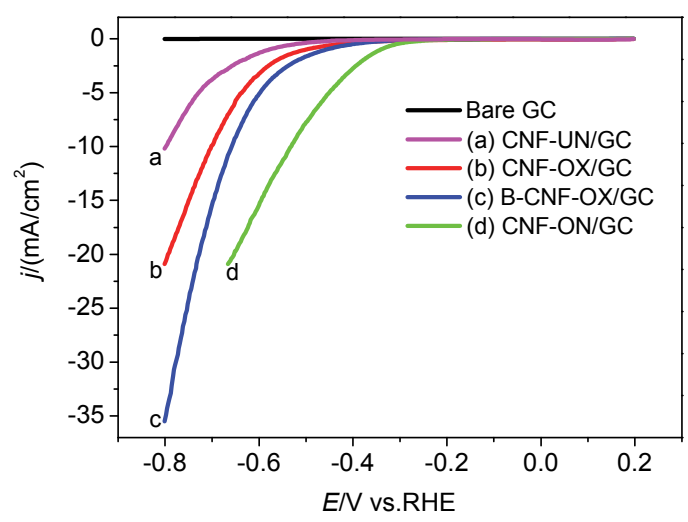

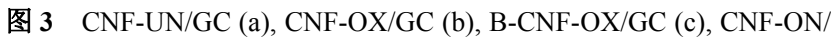
$\mathrm{GC}(\mathrm{d}) 4$ 种电极在氮气饱和的 $0.5 \mathrm{~mol} / \mathrm{L} \mathrm{H}_{2} \mathrm{SO}_{4}$ 溶液中的线性扫描伏安 曲线图, 扫描速度为 $10 \mathrm{mV} / \mathrm{s}$

Figure 3 LSV curves for HER on CNF-UN/GC (a), CNF-OX/GC (b), $\mathrm{B}-\mathrm{CNF}-\mathrm{OX} / \mathrm{GC}$ (c), CNF-ON/GC (d) electrodes in $\mathrm{N}_{2}$-saturated 0.5 $\mathrm{mol} / \mathrm{L} \mathrm{H}_{2} \mathrm{SO}_{4}$ with a scan rate of $10 \mathrm{mV} / \mathrm{s}$

C 原子的带电状态, 从而影响整个 CNFs 的电子结构, 使其表现出更高的 HER 催化活性.

同时从图 3 中还可以发现，对 CNF-OX 掺 B 处理后， 其催化活性有所增强, 经计算得 B-CNF-OX 的起始还原 过电位为 $458 \mathrm{mV}$, 相比 CNF-OX 正移 $43 \mathrm{mV}$, 两者在过 电位为 $0.8 \mathrm{~V}$ 时的阴极电流密度分别为 $35.5 \mathrm{~mA} / \mathrm{cm}^{2}$ 和 $20.9 \mathrm{~mA} / \mathrm{cm}^{2}$, 说明 B 原子的掺入对提高 HER 催化活性 有促进作用. 但我们可以发现 B-CNF-OX 的催化活性还 是要低于 $\mathrm{CNF}-\mathrm{ON}$, 这可能是由于 $\mathrm{CNF}-\mathrm{ON}$ 中吡咯型和 石墨层型 $\mathrm{N}$ 原子的掺入使得与之相邻的 $\mathrm{C}$ 原子带有更 多的正电荷，从而使 $\mathrm{C}$ 原子成为催化活性位促进了析氢 反应过程. 而对于 B-CNF-OX, 一方面是由于 $\mathrm{B}$ 原子电 负性只有 2.04, 相比 $\mathrm{N}$ 原子的 3.04, 使其在影响与之相 邻 $\mathrm{C}$ 原子的带电状态不如 $\mathrm{N}$ 原子显著; 另一方面由于 $\mathrm{B}$ 原子在 $\mathrm{B}-\mathrm{CNF}-\mathrm{OX}$ 中主要是以 $\mathrm{BC}_{2} \mathrm{O}$ 和 $\mathrm{BCO}_{2}$ 形式存在, 其对 $\mathrm{C}$ 原子带电状态影响有限, 从而使得 $\mathrm{B}-\mathrm{CNF}-\mathrm{OX}$ 催 化活性提升有限.

\subsection{CNFs 的析氢反应机理}

电解水析氢反应就是氢离子在阴极上获得电子还 原为氢原子, 最后以氢气析出的过程, 这个过程不是一 步完成的. 一般认为, 氢离子在阴极上的还原过程至少 应包括液相传质步骤、电子转移步骤、新相生成步骤三 个单元步骤, 其中电子转移步骤又可分为三个主要过 程, 其中第一个是放电步骤(即 Volmer 反应, 如 Eq. 1):

$$
\mathrm{H}_{3} \mathrm{O}^{+}+\mathrm{e}^{-} \rightarrow \mathrm{H}_{\mathrm{ads}}+\mathrm{H}_{2} \mathrm{O}
$$

其次是, 电化学脱附步骤(即 Heyrovsky 反应, 如 Eq. 2) 或复合脱附步骤(即 Tafel 反应，如 Eq. 3):

$$
\begin{aligned}
& \mathrm{H}_{\mathrm{ads}}+\mathrm{H}_{3} \mathrm{O}^{+}+\mathrm{e}^{-} \rightarrow \mathrm{H}_{2}+\mathrm{H}_{2} \mathrm{O} \\
& \mathrm{H}_{\mathrm{ads}}+\mathrm{H}_{\mathrm{ads}} \rightarrow \mathrm{H}_{2}
\end{aligned}
$$

即对于一个完整的电子转移步骤来说, 往往是上述 步骤的组合(即 Volmer-Heyrovsky 或者 Volmer-Tafel 机 
理), 并结合其它单元步骤最终生成氢气.

而我们知道 Tafel 斜率是电催化剂的固有属性, 是 由 HER 电子转移速率控制步骤所决定的. 在某些特定 的条件下，当Volmer 反应是它的控制步骤时, Tafel 斜率 应该在 $120 \mathrm{mV} / \mathrm{dec}$ 左右, 而如果是 Heyrovsky 或 Tafel 反应为速率控制步骤, Tafel 斜率分别为 30 和 $40 \mathrm{mV} / \mathrm{dec}$ 左右 ${ }^{[22]}$.

Tafel 斜率的测定是根据 Tafel 方程 ${ }^{[23]}$ :

$\eta=E_{\mathrm{e}}-E=a+b \log (-j)=(-2.3 R T / \alpha F) \log \left(j_{\mathrm{o}}\right)+$

$(2.3 R T / \alpha F) \log (-j)$

其中 $\eta$ 是过电位, $E_{\mathrm{e}}$ 是平衡电位, $j$ 是电流密度, $j_{\mathrm{o}}$ 是交换 电流密度, $b$ 就是 Tafel 斜率. 图 4 是几种催化剂修饰电 极在 $0.5 \mathrm{~mol} / \mathrm{L} \mathrm{H}_{2} \mathrm{SO}_{4}$ 溶液中的 Tafel 曲线图, 测试得到 的动力学数值见表 2 .

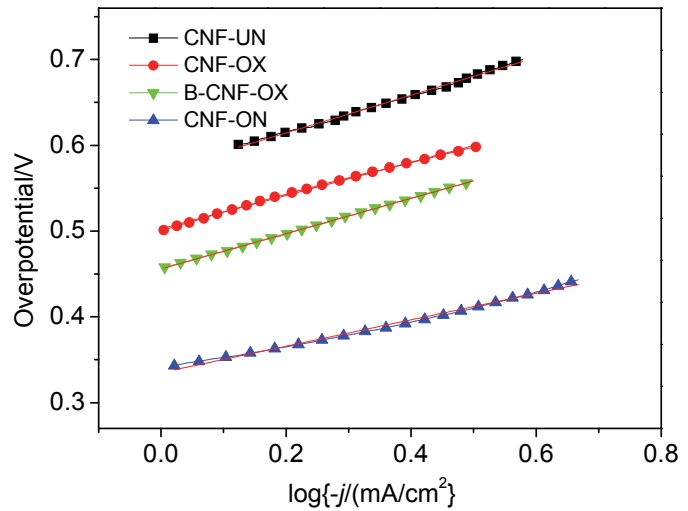

图 44 种不同的 $\mathrm{CNFs}$ 修饰电极在 $0.5 \mathrm{~mol} / \mathrm{L} \mathrm{H}_{2} \mathrm{SO}_{4}$ 溶液中的 Tafel 曲线图

Figure 4 Tafel plots of the four different CNFs modified electrodes in $0.5 \mathrm{~mol} / \mathrm{L} \mathrm{H}_{2} \mathrm{SO}_{4}$

表 24 种不同的 CNFs 修饰电极在 $0.5 \mathrm{~mol} / \mathrm{L} \mathrm{H}_{2} \mathrm{SO}_{4}$ 中的析氢动力学 参数

Table 2 Kinetic parameters of HER in $0.5 \mathrm{~mol} / \mathrm{L} \mathrm{H}_{2} \mathrm{SO}_{4}$ on the four different CNFs modified electrodes

\begin{tabular}{lccc}
\hline Samples & $b /\left({\left.\mathrm{mV} \cdot \mathrm{dec}^{-1}\right)}\right.$ & $\alpha$ & $j_{0} /\left(\mu \mathrm{A} \cdot \mathrm{cm}^{-2}\right)$ \\
\hline CNF-UN & 220 & 0.26 & 2.57 \\
CNF-OX & 193 & 0.30 & 2.56 \\
B-CNF-OX & 205 & 0.28 & 5.80 \\
CNF-ON & 154 & 0.37 & 6.68 \\
\hline
\end{tabular}

从表 2 中可以发现, 几种样品的 Tafel 斜率都超过 $120 \mathrm{mV} / \mathrm{dec}$, 因此可以认为它们催化 HER 均遵循 Volmer 反应控制, 且 CNF-ON 斜率是最小的, 而一般认 为较小的 Tafel 斜率是有利的 ${ }^{[21]}$, 因为小的斜率意味着 过电位的微小增加都会引起阴极电流密度的极大提升, 另外从交换电流密度 $j_{\mathrm{o}}$ 的数值也可以看到, 催化性能最 好的 CNF-ON 具有最大的交换电流密度值 $6.68 \mu \mathrm{A} / \mathrm{cm}^{2}$.

\section{4 电化学交流阻抗谱测试}

为了更好地了解析氢反应的诸多过程以及几种修
饰电极界面性质的差异，对几种修饰电极在不同的过电 位下进行了电化学交流阻抗谱测试，如图 5 所示.

由图 5 可以发现，几种修饰电极在三种不同过电位 下都是一个时间常数过程，即电极过程的表面反应只有 一个状态变量一一电极电位 $E$. 同时, 从图 5 中还可以 看到，对于同一修饰电极来说，随着过电位的增加，电 荷转移电阻(实轴半圆直径) 是在降低的.

另外, 从图 5 中我们还可以发现, CNF-UN 催化剂 和其它三种催化剂(CNF-OX、B-CNF-OX 和 CNF-ON) 的阻抗图有所不同. 在过电位为 $350 \mathrm{mV}$ 时, CNF-UN 的 Nyquist 图并未呈现一个完整的半圆, 这就说明相对另 外三种表面改性过的 CNFs 来说, 在同一过电位下 CNF-UN 的电荷转移电阻是最大的, 也就说明了其催化 性能是最差的.

接着我们着重研究了三种改性过的 $\mathrm{CNF}$ 修饰电极 的阻抗图谱, 这里为了定量地比较三种表面改性 CNF 修饰电极的 HER 催化性能, 我们对该过程建立了如图 6 插图的等效电路，其中 $R_{\mathrm{s}}$ 代表溶液电阻和所有的接触 电阻, CPE (Constant Phase Element)代表电极和溶液之 间界面的电双层电容, 其有两个参数: 一个是 $Y_{\mathrm{o}}$, 其量 纲是 $\Omega^{-1} \cdot \mathrm{cm}^{-2} \cdot \mathrm{s}^{-n}$; 另一个是无量纲的指数 $n, n=0$ 时, 其等效一个纯电阻 $R, n=1$ 时则等效为纯电容 $\mathrm{C}, R_{\mathrm{ct}}$ 代 表电荷转移电阻, 可以反映一个催化剂的催化性能, 在 这里是着重考察的, 对过电位为 $350 \mathrm{mV}$ 时的交流阻抗 图谱用 ZSimpWin 软件进行了拟合，拟合得到参数见表 3.

表 3 图 6 中实验测得的交流阻抗数据的模拟参数

Table 3 Model parameters simulated from the experimental EIS data in Figure 6

\begin{tabular}{lcccr}
\hline Samples & $R_{\mathrm{s}} / \Omega$ & $R_{\mathrm{ct}} / \Omega$ & $Y_{\mathrm{o}} /\left(\Omega^{-1} \cdot \mathrm{cm}^{-2} \cdot \mathrm{s}^{-n}\right)$ & $n$ \\
\hline CNF-OX & 5.034 & 4033 & 0.0005808 & 0.96 \\
B-CNF-OX & 5.867 & 3886 & 0.000716 & 0.96 \\
CNF-ON & 5.386 & 1578 & 0.0004512 & 0.97 \\
\hline
\end{tabular}

从表 3 中可以看到, 含氮官能团的 CNF-ON 相比其 它两种催化剂具有最小的电荷转移电阻 $\left(R_{\mathrm{ct}}=1578 \Omega\right)$, 即对 CNF-OX 进行掺 $\mathrm{N}$ 处理后, CNF-ON 的导电性得到 极大的增强, 从而加快了析氢反应过程的电荷转移速 率，这可归因于 $\mathrm{N}$ 的掺杂影响了其周围 $\mathrm{C}$ 原子的电荷分 布，从而影响了整个 CNF 的电子结构，带来了催化性能 的提升. 同时我们发现，对 CNF-OX 进行掺 B 处理后， B-CNF-OX 的电荷转移电阻相比 CNF-OX 也有所下降, 但不是很明显. 从 $R_{\mathrm{ct}}(\mathrm{CNF}-\mathrm{OX}>\mathrm{B}-\mathrm{CNF}-\mathrm{OX}>$ CNF-ON)大小顺序可以看出电化学交流阻抗谱(EIS)测 试结果和 LSV 测试结果一致, 即对 CNF-OX 进行掺 N 处理比对其进行掺 B 处理有更高的 HER 催化性能的提 升. 

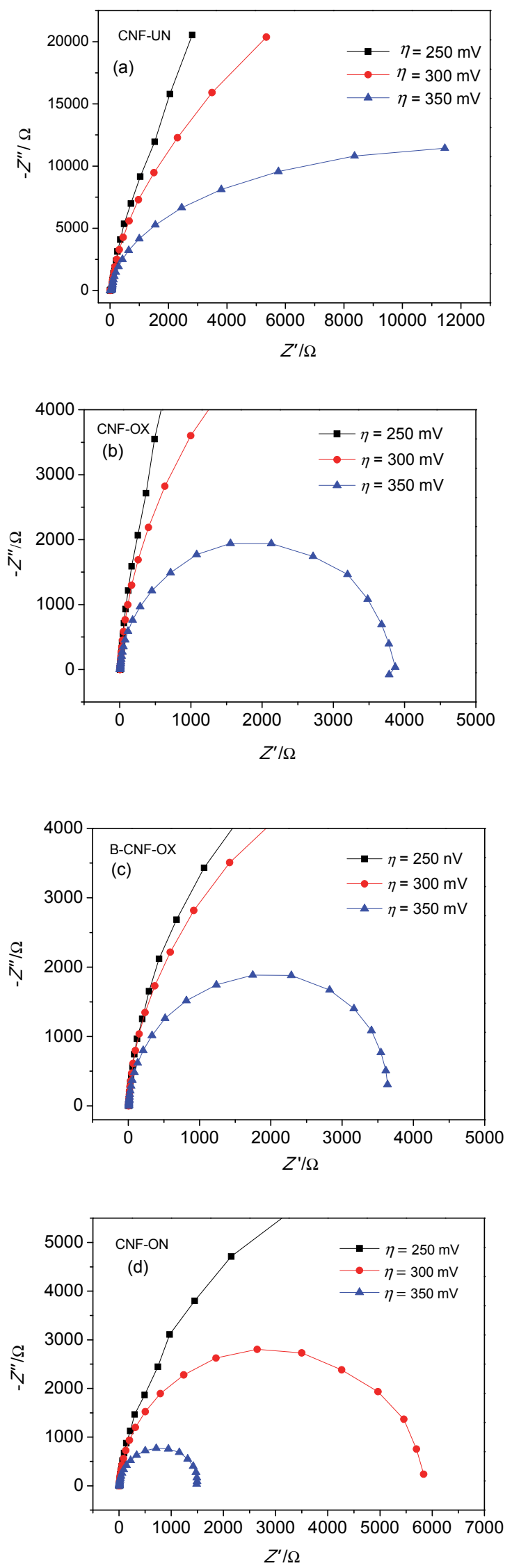

图 5 几种不同 CNFs 修饰电极在三种不同过电位下的 Nyquist 图 Figure 5 Nyquist plots of the different CNFs modified electrodes at three different overpotentials

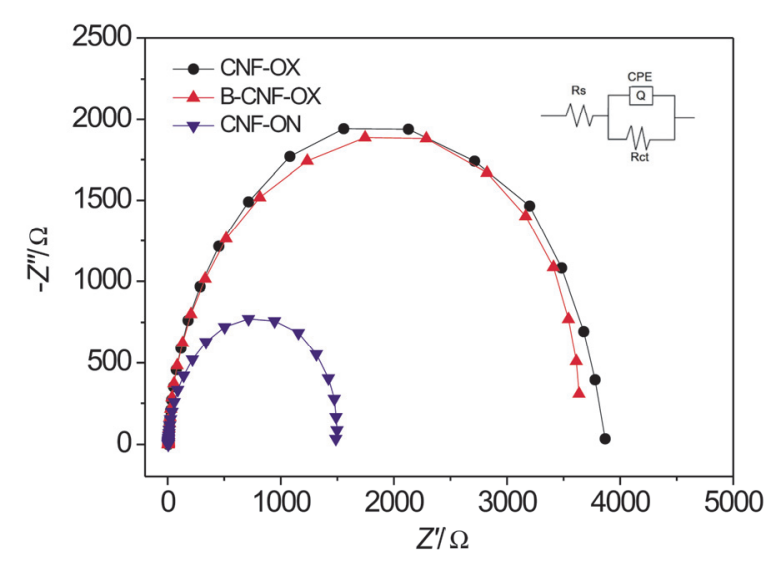

图 6 三种不同的 $\mathrm{CNF} / \mathrm{GC}$ 电极在过电位为 $350 \mathrm{mV}$ 时的 Nyquist 图 Figure 6 Nyquist plots of the three different $\mathrm{CNF} / \mathrm{GC}$ electrodes at $\eta=$ $350 \mathrm{mV}$

\section{3 结论}

采用超声处理的方法可以成功地在 CNF 表面引入 含氧和含氮官能团, 嗍酸高温热解法可以在 CNF 表面 引入 $\mathrm{B}$ 原子, LSV 和电化学交流阻抗测试结果均表明 $\mathrm{CNF}$ 表面 $\mathrm{N}$ 原子的掺入相比 $\mathrm{O}$ 和 $\mathrm{B}$ 原子的掺入对其 HER 催化性能的影响更为显著, 即 CNF-ON 表现出最 佳的 HER 催化性能. 从本文研究结果可以得到: 一是 CNFs 作为一种新型的碳材料, 其在电催化反应中有良 好的催化性能; 二是 CNFs 的表面改性对其催化析氢反 应活性有很明显的影响. 因此可以通过合理的掺杂来调 控 CNFs 的表面性质, 提高其电催化活性, 使其作为一 种非金属催化剂在电催化反应中有更广泛的应用.

\section{4 实验部分}

\section{1 原料}

本文使用的纳米碳纤维为管式纳米碳纤维 $(t-\mathrm{CNF}$, 纯度 $>95 \%$ ) 由深圳纳米港公司提供, 本文无特殊说明 CNF-UN 均指未处理过的 $t$-CNF.

\section{2 纳米碳纤维的超声处理}

首先将纳米碳纤维在混酸(浓硝酸和浓硫酸)中超声 处理以在纳米碳纤维表面引入含氧官能团 ${ }^{[24]}$, 具体如 下. 将 $1 \mathrm{~g}$ 纳米碳纤维加入含 $188 \mathrm{~mL}$ 浓硝酸、 $160 \mathrm{~mL}$ 浓硫酸和 $12 \mathrm{~mL}$ 水的混合溶液中, 在水浴超声仪中超声 氧化处理 $2 \mathrm{~h}$ 后, 过滤, 洗涤, 于 $120{ }^{\circ} \mathrm{C}$ 真空干燥 $12 \mathrm{~h}$. 经过混酸处理的纳米碳纤维标记为 CNF-OX, 接着取 $200 \mathrm{mg}$ 经过超声氧化处理的纳米碳纤维, 在 $100 \mathrm{~mL}$ 氨 水中 $60{ }^{\circ} \mathrm{C}$ 超声处理 $3 \mathrm{~h}$, 引入含氮官能团. 过滤、洗涤 及干燥步骤与前一致. 在氨水中处理的 CNF-OX 标记为 CNF-ON.

\subsection{CNF-OX 的高温掺 B 处理}

采用高温处理的方法对 CNF-OX 进行了掺 B 处 
理 ${ }^{[15]}$, 具体如下. 准确称取 $100 \mathrm{mg}$ CNF-OX 和 $2 \mathrm{~g}$ 硼酸 置于研钵中, 研磨 $30 \mathrm{~min}$ 以获得均一的混合物, 然后在 $\mathrm{N}_{2}$ 保护下以 $5{ }^{\circ} \mathrm{C} / \mathrm{min}$ 升温至 $800{ }^{\circ} \mathrm{C}$, 并维持 $4 \mathrm{~h}$, 最后 在 $\mathrm{N}_{2}$ 保护下降至室温. 接着取出热处理好的上述固体 置于 $3 \mathrm{~mol} / \mathrm{L} \mathrm{KOH}$ 溶液中回流处理 $2 \mathrm{~h}$ 以除去未反应的 含硼组分, 再经过滤、洗涤、干燥以获得掺 B 处理的 B-CNF-OX.

\subsection{CNF 修饰玻碳(GC)电极的制备}

将 CNF 催化剂超声分散在 $0.5 \mathrm{wt} \%$ 的 Nafion 溶液中 以获得均匀的黑色溶液, 浓度为 $10 \mathrm{mg} / \mathrm{mL}$. 通过移液 枪将 $5 \mu \mathrm{L}$ 上述溶液滴加在直径为 $5 \mathrm{~mm}$ 的玻碳电极上, 待溶剂蒸发后就获得 $\mathrm{CNF} / \mathrm{GC}$ 电极. 在表面修饰前, 玻 碳电极先用 $30 \mathrm{~nm}$ 的氧化铝打磨成镜面, 然后分别用丙 酮、乙醇和水清洗, 接着在超纯水中超声清洗 $5 \mathrm{~min}$, 在 空气中干燥 $30 \mathrm{~min}$. CNF-UN、CNF-OX、B-CNF-OX、 $\mathrm{CNF}-\mathrm{ON}$ 修饰的玻碳电极分别为 CNF-UN/GC、 CNF-OX/GC、B-CNF-OX/GC、CNF-ON/GC.

\section{5 催化剂表征}

$\mathrm{CNF} / \mathrm{GC}$ 催化剂的电化学测试是在 PGSTAT 302N 电化学工作站(Eco Chemie B.V., the Netherlands)上完成 的, 所用的电解质溶液为 $0.5 \mathrm{~mol} / \mathrm{L} \mathrm{H}_{2} \mathrm{SO}_{4}$ 溶液, 测试温 度为室温. 采用常规的三电极体系, 制备得到的 $\mathrm{CNF} / \mathrm{GC}$ 电极作为工作电极, 铂丝电极作为对电极, $\mathrm{Ag} / \mathrm{AgCl}(10 \mathrm{wt} \% \mathrm{KCl})$ 电极作为参比电极. 测试结果中 所有的电位均调整为相对可逆氢电极的电位(vs. RHE).

\section{References}

[1] Liu, L.; Zha, D.-W.; Wang, Y.; He, J.-B. Int. J. Hydrogen Energy 2014, 39, 14712.

[2] Cao, X.; Han, Y.; Gao, C.; Xu, Y.; Huang, X.; Willander, M.; Wang, N. Nano Energy 2014, 9, 301.
[3] Bessel, C. A.; Laubernds, K.; Rodriguez, N. M.; Baker, R. T. K. J. Phys. Chem. B 2001, 105, 1115.

[4] Aricò, A. S.; Bruce, P.; Scrosati, B.; Tarascon, J.-M.; Van Schalkwijk, W. Nat. Mater. 2005, 4, 366.

[5] Taylor, R.; Humffray, A. J. Electroanal. Chem. Interfacial Electrochem. 1975, 64, 63.

[6] Zhong, R.-S.; Qin, Y.-H.; Niu, D.-F.; Zhang, X.-S.; Zhou, X.-G.; Sun, S.-G.; Yuan, W.-K. Electrochim. Acta 2013, 89, 157.

[7] Qin, Y.-H.; Jiang, Y.; Niu, D.-F.; Zhang, X.-S.; Zhou, X.-G.; Niu, L.; Yuan, W.-K. J. Power Sources 2012, 215, 130.

[8] Qin, Y.-H.; Li, H.-C.; Yang, H.-H.; Zhang, X.-S.; Zhou, X.-G.; Niu, L.; Yuan, W.-K. J. Power Sources 2011, 196, 159.

[9] Zhang, Z.-G.; Zhao, T.-K.; Li, T.-H.; Wang, J.-B.; Guo, Z.-X. Carbon 2014, 26. (张智广, 赵廷凯, 李铁虎, 王建兵, 郭争光, 炭 素, 2014, 26.)

[10] Cao, Y.; Yu, H.; Tan, J.; Peng, F.; Wang, H.; Li, J.; Zheng, W.; Wong, N.-B. Carbon 2013, 57, 433.

[11] Zhao, D.-M.; Li, Z.-W.; Liu, L.-D.; Zhang, Y.-H.; Ren, D.-C.; Li, J. Acta Chim. Sinica 2013, 72, 185. (赵冬梅, 李振伟, 刘领弟, 张艳 红, 任德财, 李坚, 化学学报, 2013, 72, 185.)

[12] Lu, Z.-J.; Xu, M.-W.; Bao, S.-J.; Chai, H. Acta Chim. Sinica 2013, 71, 957. (鲁振江, 徐茂文, 包淑娟, 柴卉, 化学学报, 2013, 71, 957.)

[13] Qu, L.; Liu, Y.; Baek, J.-B.; Dai, L. ACS nano 2010, 4, 1321.

[14] Li, Y.; Mi, R.; Li, S.; Liu, X.; Ren, W.; Liu, H.; Mei, J.; Lau, W.-M. Int. J. Hydrogen Energy 2014, 39, 16073.

[15] Cheng, Y.; Tian, Y.; Fan, X.; Liu, J.; Yan, C. Electrochim. Acta 2014, 143, 291.

[16] Li, L.-X.; Zhao, H.-W.; Xu, W.-W.; Zhang, Y.-Q.; An, B.-G.; Geng, X. Acta Phys.-Chim. Sin. 2015, 31(3), 498. (李莉香, 赵宏伟, 许微 微, 张砚秋, 安百钢, 耿新, 物理化学学报, 2015, 31(3), 498.)

[17] Zhang, J.; Wu, S.; Chen, X.; Pan, M.; Mu, S. J. Power Sources 2014 $271,522$.

[18] Ratso, S.; Kruusenberg, I.; Vikkisk, M.; Joost, U.; Shulga, E.; Kink, I.; Kallio, T.; Tammeveski, K. Carbon 2014, 73, 361.

[19] Zhong, R.-S.; Qin, Y.-H.; Niu, D.-F.; Tian, J.-W.; Zhang, X.-S.; Zhou, X.-G.; Sun, S.-G.; Yuan, W.-K. J. Power Sources 2013, 225, 192.

[20] Moncoffre, N.; Hollinger, G.; Jaffrezic, H.; Marest, G.; Tousset, J. Nucl. Instrum. Methods Phys. Res., Sect. B 1985, 7, 177.

[21] Figueiredo, J. L.; Pereira, M. F. R. Catal. Today 2010, 150, 2.

[22] Xie, J.; Zhang, J.; Li, S.; Grote, F.; Zhang, X.; Zhang, H.; Wang, R.; Lei, Y.; Pan, B.; Xie, Y. J. Am. Chem. Soc. 2013, 135, 17881.

[23] Liu, B.; He, J.-B.; Chen, Y.-J.; Wang, Y.; Deng, N. Int. J. Hydrogen Energy 2013, 38, 3130.

[24] Jiang, Y.; Zhang, J.; Qin, Y.-H.; Niu, D.-F.; Zhang, X.-S.; Niu, L.; Zhou, X.-G.; Lu, T.-H.; Yuan, W.-K. J. Power Sources 2011, 196, 9356.

(Qin, X.) 